\title{
Hypertranscendence and algebraic independence of certain infinite products
}

\author{
by \\ Peter Bundschuh (Köln) and KeiJo VÄÄnänen (Oulu)
}

To Rob Tijdeman on his 75th birthday

1. Introduction and results. Let $d \geq 2$ always denote a fixed integer. If $p(z)$ is a polynomial with complex coefficients satisfying $p(0)=1$, then the infinite product

$$
F(z)=\prod_{j=0}^{\infty} p\left(z^{d^{j}}\right)
$$

is analytic in $\mathbb{D}:=\{z \in \mathbb{C}:|z|<1\}$ and satisfies the simple Mahler type functional equation

$$
w(z)=p(z) w\left(z^{d}\right) .
$$

The solutions of such functional equations are either rational or transcendental. If a rational function $a(z) / b(z)$ with coprime non-zero polynomials $a(z)$ and $b(z)$ satisfies $(2)$, then

$$
a(z) b\left(z^{d}\right)=p(z) b(z) a\left(z^{d}\right) .
$$

Since $a(z)$ and $b(z)$ are coprime, it follows that $a\left(z^{d}\right) \mid a(z)$ giving $a(z)=c \in \mathbb{C}^{\times}$. Thus $p(z)=$ $b\left(z^{d}\right) / b(z)$. On the other hand, if $p(z)$ is of this form, then $F(z)=1 / b(z)$ is a rational function. Further, if $b(z) \mid b\left(z^{d}\right)$, then all zeros of $b(z)$ lie on the unit circle. An important class of infinite products of cyclotomic polynomials is considered in [6] and [3]. In particular, [3, Theorem 1.1] states that $F(z)$ with $p(z)=\Phi_{\ell}(z)$, the $\ell$ th cyclotomic polynomial (with the convention $\Phi_{1}(z):=1-z$ ), is hypertranscendental if and only if $d$ is either composite or a prime not dividing $\ell$. Recall that a function $f(z)$ is said to be hypertranscendental, if $f(z)$ and all derivatives $f^{(\ell)}(z)(\ell \geq 1)$ are algebraically independent over $\mathbb{C}(z)$. In [3], we also studied algebraic independence of infinite products with different $\Phi_{\ell}(z)$.

In our present work, we are interested in infinite products (1), where $p(z)$ has at least one zero not lying on the unit circle, or equivalently, with $p^{-1}(0) \not \subset \partial \mathbb{D}$. As mentioned above, $F(z)$ is a transcendental function in this case, and our first result yields a condition for its hypertranscendence.

To suitably formulate this result, we note that the hypothesis $p^{-1}(0) \not \subset \partial \mathbb{D}$ allows the following alternative: If $p^{-1}(0) \cap \mathbb{D} \neq \emptyset$, then let $\omega$ denote an element from this set of minimal absolute value, or if $p^{-1}(0) \cap \mathbb{D}=\emptyset$, then let $\Omega$ denote an element from $p^{-1}(0)$ of maximal absolute value. Clearly $|\omega|<1$ or $|\Omega|>1$.

TheOREM 1.1. Let $p \in \mathbb{C}[z]$ satisfy $p(0)=1$ and $p^{-1}(0) \not \subset \partial \mathbb{D}$. If $|\omega|<1$ (or $|\Omega|>1$ ) and, for each integer $j \geq 1$, not all solutions of $z^{d^{j}}=\omega$ (or of $z^{d^{j}}=\Omega$ ) are zeros of the product $p\left(z^{d^{j-1}}\right) \cdot \ldots \cdot p(z)$, then the function $F(z)$ in (1) is hypertranscendental.

Under the conditions on $p(z)$ in Theorem 1.1, let $\left\{\alpha_{1}, \ldots, \alpha_{\nu}\right\}:=p^{-1}(0) \backslash \partial \mathbb{D}$, and denote by $n\left(\alpha_{\kappa}\right)$ and $N\left(\alpha_{\kappa}\right)$ the number of $\alpha_{k}$ such that $\left|\alpha_{\kappa}\right|=\left|\alpha_{k}\right|$ and $\left|\alpha_{\kappa}\right|=\left|\alpha_{k}\right|^{d^{j}}$ for some integer $j \geq 1$, respectively. Assume that $\left|\alpha_{1}\right| \leq \cdots \leq\left|\alpha_{\nu_{1}}\right|<1<\left|\alpha_{\nu_{1}+1}\right| \leq \cdots \leq\left|\alpha_{\nu}\right|$. Then $N\left(\alpha_{1}\right) \leq \nu_{1}-n\left(\alpha_{1}\right)$, if $\left|\alpha_{1}\right|<1$, and $N\left(\alpha_{\nu}\right) \leq \nu-\nu_{1}-n\left(\alpha_{\nu}\right)$, if $\left|\alpha_{\nu}\right|>1$. Keeping the notations for $\omega$ and $\Omega$ as introduced before Theorem 1.1 , this result implies the following.

Corollary 1.2. If $|\omega|<1$ and $N(\omega)<d$, or if $|\Omega|>1$ and $N(\Omega)<d$, then the function $F(z)$ is hypertranscendental. 
In particular, if $|\omega|<1$ and $|\omega| \neq\left|\alpha_{k}\right|^{d^{j}}$ for any pair of integers $(j, k)$ with $j \geq 1$ and $1 \leq k \leq \nu_{1}$, then $N(\omega)=0$ and Corollary 1.2 applies. We also have $N(\omega) \leq 1$ or $N(\Omega) \leq 1$, if $\nu=2$. If all $\alpha_{i}$ are real, then the following more explicit result holds.

Corollary 1.3. In the case $\left\{\alpha_{1}, \ldots, \alpha_{\nu}\right\} \subset \mathbb{R}$, the function $F(z)$ is hypertranscendental if either $d \geq 3$, or $d=2$ and $-\alpha_{i} \notin\left\{\alpha_{1}, \ldots, \alpha_{\nu}\right\}$ for each $i=1, \ldots, \nu$.

The proof of Theorem 1.1 is based on a hypertranscendence criterion for degree 1 Mahler functions by Ke. Nishioka [8]. There is also a criterion of Kubota [7] for the consideration of the algebraic independence of products

$$
F_{i}(z)=\prod_{j=0}^{\infty} p_{i}\left(z^{d^{j}}\right) \quad(i=1, \ldots, n)
$$

with different polynomials $p_{i}(z)$ satisfying the properties of $p(z)$ in Theorem 1.1. Apparently, the question on the algebraic independence of different functions and their derivatives have so far only been rarely investigated in the literature. In this connection, we would like to point out recent remarkable progress in the consideration of algebraic independence of some higher degree Mahler functions and their derivatives, see [1] and [5].

Our main purpose here is to study algebraic independence of the functions $F_{i}(z)$ and their first derivatives satisfying a system of Mahler type functional equations

$$
F_{i}(z)=p_{i}(z) F_{i}\left(z^{d}\right), F_{i}^{\prime}(z)=p_{i}^{\prime}(z) F_{i}\left(z^{d}\right)+d z^{d-1} p_{i}(z) F_{i}^{\prime}\left(z^{d}\right)
$$

with $p_{i}(0)=1$ and $p_{i}^{-1}(0) \not \subset \partial \mathbb{D}$ for $i=1, \ldots, n$. To state our result, we denote

$$
v_{i}(z):=\prod_{\alpha \in p_{i}^{-1}(0) \backslash \partial \mathbb{D}}\left(1-\frac{z}{\alpha}\right)^{\mu(\alpha)}
$$

where $\mu(\alpha)$ is the zero multiplicity of $\alpha$ in $p_{i}(z)$.

TheOREM 1.4. Assume that the polynomials $p_{i}(z)$ satisfy $p_{i}(0)=1$ and $p_{i}^{-1}(0) \not \subset \partial \mathbb{D}$ for $i=1, \ldots, n$, and that no two of the corresponding polynomials $v_{i}(z)$ have a common zero. Moreover, suppose for any zero $\alpha$ of $\prod_{i=1}^{n} v_{i}(z)$ and for each integer $j \geq 1$, that not all solutions of $z^{d^{j}}=\alpha$ are zeros of $\prod_{i=1}^{n} v_{i}\left(z^{d^{j-1}}\right) \cdot \ldots \cdot v_{i}(z)$. Then the functions $F_{1}(z), F_{1}^{\prime}(z), \ldots, F_{n}(z), F_{n}^{\prime}(z)$ are algebraically independent over $\mathbb{C}(z)$.

As an immediate consequence of Theorem 1.4, we present the following result on the functions

$$
w_{i}(z):=\frac{F_{i}^{\prime}(z)}{F_{i}(z)}=\sum_{j=0}^{\infty} \frac{d^{j} z^{d^{j}-1} p_{i}^{\prime}\left(z^{d^{j}}\right)}{p_{i}\left(z^{d^{j}}\right)} \quad(i=1, \ldots, n)
$$

satisfying

$$
d z^{d-1} w_{i}\left(z^{d}\right)=w_{i}(z)-\frac{p_{i}^{\prime}(z)}{p_{i}(z)} \quad(i=1, \ldots, n) .
$$

Corollary 1.5. Under the assumptions of Theorem 1.4 the functions $w_{1}(z), \ldots, w_{n}(z)$ are algebraically independent over $\mathbb{C}(z)$.

To give some more corollaries to Theorem 1.4, we now define $\prod_{i=1}^{n} v_{i}(z)=: p(z)$ and use the notations introduced before Corollary 1.2. Then $N\left(\alpha_{i}\right) \leq \min \left\{\nu_{1}-i, \nu_{1}-n\left(\alpha_{i}\right)\right\}$ if $\left|\alpha_{i}\right|<1$, and 
$N\left(\alpha_{i}\right) \leq \min \left\{i-\nu_{1}, \nu-\nu_{1}-n\left(\alpha_{i}\right)\right\}$ if $\left|\alpha_{i}\right|>1$. By Theorem 1.4, we now have the following results.

Corollary 1.6. If $\max \left\{N\left(\alpha_{i}\right)\right\}<d$, then the functions $F_{1}(z), F_{1}^{\prime}(z), \ldots, F_{n}(z), F_{n}^{\prime}(z)$ are algebraically independent over $\mathbb{C}(z)$.

Corollary 1.7. If $v(z)=1+z / a$ with $a \in \mathbb{C}^{\times} \backslash \partial \mathbb{D}$ and if $v_{i}(z):=v\left(\rho_{i} z\right)$ with distinct $\rho_{1}, \ldots, \rho_{n} \in$ $\partial \mathbb{D}$, then the functions $F_{1}(z), F_{1}^{\prime}(z), \ldots, F_{n}(z), F_{n}^{\prime}(z)$ are algebraically independent over $\mathbb{C}(z)$.

If $v(z)=c\left(z-\alpha_{1}\right)\left(z-\alpha_{2}\right)$ with a constant $c \neq 0$ and $\alpha_{1}, \alpha_{2} \in \mathbb{C}^{\times} \backslash \partial \mathbb{D}$, then $N:=\max \left\{N\left(\alpha_{1}\right), N\left(\alpha_{2}\right)\right\}$ $\leq 1$ for the polynomial $p(z)=v(z)$.

COROllary 1.8. Let $v(z)=c\left(z-\alpha_{1}\right)\left(z-\alpha_{2}\right)$ be as above, and define $v_{i}(z):=v\left(\rho_{i} z\right)$ with distinct $\rho_{1}, \ldots, \rho_{n} \in \partial \mathbb{D}$. If $N=0$, or $N=1$ and $n<d$, then the functions $F_{1}(z), F_{1}^{\prime}(z), \ldots, F_{n}(z), F_{n}^{\prime}(z)$ are algebraically independent over $\mathbb{C}(z)$.

For example, if we choose $v(z)=1+z-z^{2}$, then above $N=0$ and Corollary 1.8 applies. For the very special case $d=2$ and $\rho_{1}=1, \rho_{2}=-1$ see also [4, Theorem 1.1].

Let now $v_{i}(z)=1+z / a_{i}(i=1, \ldots, n)$ with distinct $a_{1}, \ldots, a_{n} \in \mathbb{C}^{\times}$satisfying $\left|a_{1}\right| \leq \cdots \leq\left|a_{n_{1}}\right|<$ $1<\left|a_{n_{1}+1}\right| \leq \cdots \leq\left|a_{n}\right|$. Assume that, for each integer $j \geq 1,\left|a_{i}\right| \neq\left.\left|a_{k}\right|\right|^{j}\left(1 \leq i<k \leq n_{1}\right)$ and $\left|a_{i}\right|^{d^{j}} \neq\left|a_{k}\right|\left(n_{1}+1 \leq i<k \leq n\right)$.

Corollary 1.9. Let the polynomials $v_{i}(z)=1+z / a_{i}(i=1, \ldots, n)$ be as above. Then the functions $F_{1}(z), F_{1}^{\prime}(z), \ldots, F_{n}(z), F_{n}^{\prime}(z)$ are algebraically independent over $\mathbb{C}(z)$.

The following analogue of Corollary 1.3 shows that all of the above restrictions are not needed if all $a_{i}$ are real.

Corollary 1.10. In the case $\left\{\alpha_{1}, \ldots, \alpha_{\nu}\right\} \subset \mathbb{R}$, the functions $F_{1}(z), F_{1}^{\prime}(z), \ldots, F_{n}(z), F_{n}^{\prime}(z)$ are algebraically independent over $\mathbb{C}(z)$ if either $d \geq 3$, or $d=2$ and $-\alpha_{i} \notin\left\{\alpha_{1}, \ldots, \alpha_{\nu}\right\}$ for each $i=1, \ldots, \nu$.

In our next result, we consider algebraic independence of the functions (3) and the functions studied in [3], namely infinite products of cyclotomic polynomials

$$
\mathcal{F}_{\ell}(z):=\prod_{j=0}^{\infty} \Phi_{\ell}\left(z^{d^{j}}\right) \quad \text { with integers } \ell \geq 1 \text { prime to } d .
$$

TheOREm 1.11. Assume that the polynomials $p_{i}(z)$ satisfy the assumptions of Theorem 1.4. Then the functions (3) and (5) are algebraically independent over $\mathbb{C}(z)$.

As an example for this theorem, we give the algebraic independence of the functions $F_{i}(z)(i=$ $1, \ldots, n)$ with $d=2$ and $p_{i}(z)=v\left(\rho_{i} z\right)$, where $v(z)=1+z-z^{2}$ and $\rho_{i}$ are as above, and $\mathcal{F}_{\ell}(z)$ with odd integers $\ell$. Here $\mathcal{F}_{1}(z)$ is the generating power series of the Thue-Morse sequence on $\{-1,1\}$ and $\mathcal{F}_{3}(z)$ is the generating power series of the Stern diatomic sequence, and so this result generalizes [4, Theorem 1.1] and partly also [3, Theorem 1.3].

2. Proof of Theorem 1.1 and of Corollary 1.3. By using the hypertranscendence criterion of Ke. Nishioka [8], it is proved in [2] that, to establish Theorem 1.1, it is enough to show that the functional equation

$$
w(z)-d w\left(z^{d}\right)=\frac{z p^{\prime}(z)}{p(z)}
$$


has no solution $w(z) \in \mathbb{C}(z)$. Assume now that (6) has a rational solution $w(z)$. Further, let us assume that $|\omega|<1$ (the case $|\Omega|>1$ is similar). Since the right-hand side of (6) has a pole at $\omega, w(z)$ must have poles $\alpha$ with $0<|\alpha|<1$. Let $\alpha_{0}$ be such a pole with minimal absolute value. If $\left|\alpha_{0}\right|<|\omega|$, then we get a contradiction, since $\left|\alpha_{0}^{d}\right|<\left|\alpha_{0}\right|$. Also $\left|\omega^{d}\right|<|\omega|$, and therefore $\omega$ is a pole of $w(z)$. By our assumption, there exists a solution $\alpha_{1}$ of $z^{d}=\omega$, which is not a pole of the right-hand side of (6). Thus, $\alpha_{1}$ is a pole of $w(z)$. By $(6)$,

$$
w(z)-d^{2} w\left(z^{d^{2}}\right)=\frac{z p^{\prime}(z)}{p(z)}+\frac{d z^{d} p^{\prime}\left(z^{d}\right)}{p\left(z^{d}\right)} .
$$

Using our assumptions again, we see that there exists a solution $\alpha_{2}$ of $z^{d^{2}}=\omega$, which is a pole of $w(z)$. Continuing in this way, we get a final contradiction proving Theorem 1.1.

To prove Corollary 1.2, we just note that the conditions of this corollary imply the assumptions of Theorem 1.1 .

Proof of Corollary 1.3. Let us assume $\left\{\alpha_{1}, \ldots, \alpha_{\nu}\right\} \subset \mathbb{R}$, and consider the solutions of $z^{d^{j}}=\alpha \in$ $\left\{\alpha_{1}, \ldots, \alpha_{\nu}\right\}$. In particular, we may choose $\alpha=\omega$ or $\alpha=\Omega$. There exists a root with an argument $2 \pi / d^{j}$ if $\alpha>0$, or $\pi / d^{j}$ if $\alpha<0$. If $d \geq 3$, then none of the roots of $z^{d^{k}}=\alpha_{i}(0 \leq k<j, 1 \leq i \leq \nu)$ has such an argument. Therefore, the condition of Theorem 1.1 is satisfied if $d \geq 3$.

The equation $z^{2^{j}}=\alpha$ has $2^{j}$ solutions all having absolute value $\sqrt[2^{j}]{|\alpha|}$. For all $k=0, \ldots, j-1$, the solutions of $z^{2^{k}}=\alpha_{i}$ can have this absolute value only if $\left|\alpha_{i}\right|=\sqrt[2^{j-k}]{|\alpha|}$. By our hypothesis, either $\alpha_{i}=\sqrt[2^{j-k}]{|\alpha|}$ or $-\alpha_{i}=\sqrt[2^{j-k}]{|\alpha|}$ is possible. This means that among the solutions of $z^{2^{k}}=\alpha_{i}(i=1, \ldots, \nu)$ there are at most $2^{k}$ solutions of $z^{2^{j}}=\alpha$. Thus, this last equation has at least one root which is not a zero of $p\left(z^{2^{j-1}}\right) \cdot \ldots \cdot p(z)$ proving Corollary 1.3.

3. Proof of Theorem 1.4. To prepare this proof, we first note that

$$
U_{i}(z) F_{i}\left(z^{d}\right)=d z^{d-1} p_{i}(z) F_{i}(z), \quad U_{i}(z) F_{i}^{\prime}\left(z^{d}\right)=-p_{i}^{\prime}(z) F_{i}(z)+p_{i}(z) F_{i}^{\prime}(z)
$$

for $i=1, \ldots, n$, where $U_{i}(z):=d z^{d-1} p_{i}^{2}(z)$. Let now $P \neq 0$ be a polynomial

$$
P\left(z, x_{1}, y_{1}, \ldots, x_{n}, y_{n}\right)=\sum_{\underline{m}_{n}} p_{\underline{m}_{n}}(z) \prod_{i=1}^{n} x_{i}^{\ell_{i}} y_{i}^{k_{i}}
$$

where $\underline{m}_{n}:=\left(\ell_{1}, k_{1}, \ldots, \ell_{n}, k_{n}\right)$, and let $M$ denote the maximal value of $\left|\underline{m}_{n}\right|:=\ell_{1}+k_{1}+\cdots+\ell_{n}+k_{n}$ with $p_{\underline{m}_{n}}(z) \neq 0$. If $P\left(z, F_{1}(z), F_{1}^{\prime}(z), \ldots, F_{n}(z), F_{n}^{\prime}(z)\right)=0$, then (7) implies that also the polynomial

$$
Q\left(z, x_{1}, y_{1}, \ldots, x_{n}, y_{n}\right)=\sum_{\underline{m}_{n}} Q_{\underline{m}_{n}}\left(z, x_{1}, y_{1}, \ldots, x_{n}, y_{n}\right)
$$

where

$$
Q_{\underline{m}_{n}}\left(z, x_{1}, y_{1}, \ldots, x_{n}, y_{n}\right)=p_{\underline{m}_{n}}\left(z^{d}\right) \prod_{i=1}^{n}\left\{U_{i}(z)^{M-\ell_{i}-k_{i}}\left(d z^{d-1} p_{i}(z) x_{i}\right)^{\ell_{i}}\left(-p_{i}^{\prime}(z) x_{i}+p_{i}(z) y_{i}\right)^{k_{i}}\right\}
$$

satisfies $Q\left(z, F_{1}(z), F_{1}^{\prime}(z), \ldots, F_{n}(z), F_{n}^{\prime}(z)\right)=0$.

We first use induction on $n$ to establish the following lemma.

LEMMA 3.1. If $V(z)$ and $T(z)$ are non-zero polynomials such that $T(z)$ is a factor of the polynomial $V(z) Q\left(z, x_{1}, y_{1}, \ldots, x_{n}, y_{n}\right)$, then

$$
T(z) \mid V(z) p_{\underline{m}_{n}}\left(z^{d}\right) \prod_{i=1}^{n} U_{i}(z)^{M}
$$


holds for all $\underline{m}_{n}$.

Proof. In the case $n=1$,

$$
P\left(z, x_{1}, y_{1}\right)=\sum_{\underline{m}_{1}} p_{\underline{m}_{1}}(z) x_{1}^{\ell_{1}} y_{1}^{k_{1}}=\sum_{n_{1}=0}^{M} \sum_{k_{1}=0}^{n_{1}} p_{n_{1}-k_{1}, k_{1}}(z) x_{1}^{n_{1}-k_{1}} y_{1}^{k_{1}},
$$

and

$$
\begin{aligned}
Q\left(z, x_{1}, y_{1}\right) & =\sum_{n_{1}=0}^{M} \sum_{k_{1}=0}^{n_{1}} p_{n_{1}-k_{1}, k_{1}}\left(z^{d}\right) U_{1}(z)^{M-n_{1}}\left(d z^{d-1} p_{1}(z) x_{1}\right)^{n_{1}-k_{1}}\left(-p_{1}^{\prime}(z) x_{1}+p_{1}(z) y_{1}\right)^{k_{1}} \\
& =\sum_{n_{1}=0}^{M} \sum_{k_{1}=0}^{n_{1}} q_{n_{1}-k_{1}, k_{1}}(z) x_{1}^{n_{1}-k_{1}} y_{1}^{k_{1}}
\end{aligned}
$$

where

(9) $\quad q_{n_{1}-k_{1}, k_{1}}(z):=U_{1}(z)^{M-n_{1}} \sum_{j_{1}=k_{1}}^{n_{1}}\left(\begin{array}{l}j_{1} \\ k_{1}\end{array}\right) p_{n_{1}-j_{1}, j_{1}}\left(z^{d}\right)\left(d z^{d-1}\right)^{n_{1}-j_{1}} p_{1}(z)^{n_{1}-j_{1}+k_{1}}\left(-p_{1}^{\prime}(z)\right)^{j_{1}-k_{1}}$.

Since $T(z) \mid V(z) Q\left(z, x_{1}, y_{1}\right)$, we have $T(z) \mid V(z) q_{n_{1}-k_{1}, k_{1}}(z)$ for $0 \leq k_{1} \leq n_{1} \leq M$. For a given $n_{1}$, we obtain from (9),

$$
q_{0, n_{1}}(z)=U_{1}(z)^{M-n_{1}} p_{0, n_{1}}\left(z^{d}\right) p_{1}(z)^{n_{1}},
$$

hence

$$
T(z) \mid V(z) p_{0, n_{1}}\left(z^{d}\right) U_{1}(z)^{M-n_{1}} p_{1}(z)^{n_{1}} .
$$

Further,

$$
q_{1, n_{1}-1}(z)=U_{1}(z)^{M-n_{1}}\left\{p_{1, n_{1}-1}\left(z^{d}\right) d z^{d-1} p_{1}(z)^{n_{1}}+n_{1} p_{0, n_{1}}\left(z^{d}\right) p_{1}(z)^{n_{1}-1}\left(-p_{1}^{\prime}(z)\right)\right\},
$$

and therefore

$$
T(z) \mid V(z) p_{1, n_{1}-1}\left(z^{d}\right) U_{1}(z)^{M-n_{1}} d z^{d-1} p_{1}(z)^{n_{1}+1} .
$$

By continuing to apply formula (9) in the same way, we obtain

$$
T(z) \mid V(z) p_{j, n_{1}-j}\left(z^{d}\right) U_{1}(z)^{M-n_{1}}\left(d z^{d-1}\right)^{j} p_{1}(z)^{n_{1}+j}
$$

for $j=0,1, \ldots, n_{1}$. This gives the validity of Lemma 3.1 for $n=1$.

Assume now that Lemma 3.1 holds, if $n(\geq 2)$ is replaced by $n-1$. If $\operatorname{deg}_{x_{n}, y_{n}} P=L_{n}(\leq M)$, then we may write

$$
P\left(z, x_{1}, y_{1}, \ldots, x_{n}, y_{n}\right)=\sum_{\mu_{n}=0}^{L_{n}} \sum_{k_{n}=0}^{\mu_{n}}\left(\sum_{\underline{m}_{n-1}} p_{\underline{m}_{n-1}, \mu_{n}-k_{n}, k_{n}}(z) \prod_{i=1}^{n-1} x_{i}^{\ell_{i}} y_{i}^{k_{i}}\right) x_{n}^{\mu_{n}-k_{n}} y_{n}^{k_{n}},
$$

and correspondingly

$$
\begin{aligned}
Q\left(z, x_{1}, y_{1}, \ldots, x_{n}, y_{n}\right) & =\sum_{\mu_{n}=0}^{L_{n}} \sum_{k_{n}=0}^{\mu_{n}} q_{\mu_{n}-k_{n}, k_{n}}\left(z, x_{1}, y_{1}, \ldots, x_{n-1}, y_{n-1}\right) U_{n}(z)^{M-\mu_{n}} \\
& \times\left(d z^{d-1} p_{n}(z) x_{n}\right)^{\mu_{n}-k_{n}}\left(-p_{n}^{\prime}(z) x_{n}+p_{n}(z) y_{n}\right)^{k_{n}},
\end{aligned}
$$


where $q_{\mu_{n}-k_{n}, k_{n}}\left(z, x_{1}, y_{1}, \ldots, x_{n-1}, y_{n-1}\right)$ is a $Q$-polynomial arising from the sum

$$
\sum_{\underline{m}_{n-1}} p_{\underline{m}_{n-1}, \mu_{n}-k_{n}, k_{n}}(z) \prod_{i=1}^{n-1} x_{i}^{\ell_{i}} y_{i}^{k_{i}} .
$$

This yields

$$
\begin{aligned}
Q\left(z, x_{1}, y_{1}, \ldots, x_{n}, y_{n}\right) & =\sum_{\mu_{n}=0}^{L_{n}} U_{n}(z)^{M-\mu_{n}} \sum_{k_{n}=0}^{\mu_{n}}\left(\sum_{j_{n}=k_{n}}^{\mu_{n}}\left(\begin{array}{l}
j_{n} \\
k_{n}
\end{array}\right) q_{\mu_{n}-j_{n}, j_{n}}\left(z, x_{1}, y_{1}, \ldots, x_{n-1}, y_{n-1}\right)\right. \\
& \left.\times\left(d z^{d-1}\right)^{\mu_{n}-j_{n}} p_{n}(z)^{\mu_{n}-j_{n}+k_{n}}\left(-p_{n}^{\prime}(z)\right)^{j_{n}-k_{n}}\right) x_{n}^{\mu_{n}-k_{n}} y_{n}^{k_{n}} .
\end{aligned}
$$

Since $T(z)$ is a factor of $V(z) Q\left(z, x_{1}, y_{1}, \ldots, x_{n}, y_{n}\right)$, it is also a factor of the product

$$
\begin{aligned}
& V(z) U_{n}(z)^{M-\mu_{n}} \\
& \quad \times\left(\sum_{j_{n}=k_{n}}^{\mu_{n}}\left(\begin{array}{l}
j_{n} \\
k_{n}
\end{array}\right) q_{\mu_{n}-j_{n}, j_{n}}\left(z, x_{1}, y_{1}, \ldots, x_{n-1}, y_{n-1}\right)\left(d z^{d-1}\right)^{\mu_{n}-j_{n}} p_{n}(z)^{\mu_{n}-j_{n}+k_{n}}\left(-p_{n}^{\prime}(z)\right)^{j_{n}-k_{n}}\right)
\end{aligned}
$$

for all $\mu_{n}, k_{n}$. If $k_{n}=\mu_{n}$, we get

$$
T(z) \mid V(z) U_{n}(z)^{M-\mu_{n}} q_{0, \mu_{n}}\left(z, x_{1}, y_{1}, \ldots, x_{n-1}, y_{n-1}\right) p_{n}(z)^{\mu_{n}} .
$$

The case $k_{n}=\mu_{n}-1$ leads to

$$
\begin{aligned}
T(z) \mid V(z) U_{n}(z)^{M-\mu_{n}} & \left(q_{1, \mu_{n}-1}\left(z, x_{1}, y_{1}, \ldots, x_{n-1}, y_{n-1}\right) d z^{d-1} p_{n}(z)^{\mu_{n}}\right. \\
& \left.+\mu_{n} q_{0, \mu_{n}}\left(z, x_{1}, y_{1}, \ldots, x_{n-1}, y_{n-1}\right) p_{n}(z)^{\mu_{n}-1}\left(-p_{n}^{\prime}(z)\right)\right)
\end{aligned}
$$

implying

$$
T(z) \mid V(z) U_{n}(z)^{M-\mu_{n}} q_{1, \mu_{n}-1}\left(z, x_{1}, y_{1}, \ldots, x_{n-1}, y_{n-1}\right) d z^{d-1} p_{n}(z)^{\mu_{n}+1} .
$$

By continuing analogously, we obtain for $j=0,1, \ldots, \mu_{n}$

$$
T(z) \mid V(z) U_{n}(z)^{M-\mu_{n}} q_{j, \mu_{n}-j}\left(z, x_{1}, y_{1}, \ldots, x_{n-1}, y_{n-1}\right)\left(d z^{d-1}\right)^{j} p_{n}(z)^{\mu_{n}+j} .
$$

Thus, we have proved the divisibility relation

$$
T(z) \mid V(z) U_{n}(z)^{M} q_{\mu_{n}-k_{n}, k_{n}}\left(z, x_{1}, y_{1}, \ldots, x_{n-1}, y_{n-1}\right) \quad \text { for } 0 \leq k_{n} \leq \mu_{n} \leq L_{n} .
$$

By applying our induction hypothesis to the $Q$-polynomials $q_{\mu_{n}-k_{n}, k_{n}}\left(z, x_{1}, y_{1}, \ldots, x_{n-1}, y_{n-1}\right)$, we obtain the validity of Lemma 3.1.

For the following considerations, we recall [4, Lemma 2.2] of which we include here a short proof for the reader's convenience.

Lemma 3.2. Let $R \in \mathbb{C}(z)$ have zeros or poles in $0<|z|<1$ or in $1<|z|<+\infty$, where $\omega$ and $\Omega$ are zeros or poles of minimal and of maximal absolute value, respectively. If $|\omega|<1$ (or $|\Omega|>1$ ), assume that, for each integer $j \geq 1$, not all solutions of $z^{d^{j}}=\omega$ (or of $z^{d^{j}}=\Omega$ ) are zeros or poles of the product $R\left(z^{d^{j-1}}\right) \cdot \ldots \cdot R(z)$. Then the functional equation

$$
r(z)=r\left(z^{d}\right) R(z)
$$

has no rational solution $r \neq 0$. 
Proof. Suppose, on the contrary, that (10) has a rational solution $r(z) \neq 0$. Clearly, this $r(z)$ has no zero or pole in $|z|<|\omega|$ (or in $|z|>|\Omega|$ ). Since $\left|\omega^{d}\right|<|\omega|$ (or $\left|\Omega^{d}\right|>|\Omega|$ ) we know that $\omega^{d}$ (or $\Omega^{d}$ ) is not a zero or pole of $r(z)$, whence $\omega$ (or $\Omega$ ) is a zero or pole of $r(z)$, by (10) and a hypothesis on $R$.

On iterating (10), we conclude that $r$ satisfies

$$
r(z)=r\left(z^{d^{j}}\right) R\left(z^{d^{j-1}}\right) \cdot \ldots \cdot R(z)
$$

for any $j=1,2, \ldots$. For any such $j$, we consider all $d^{j}$ solutions of $z^{d^{j}}=\omega$ (or of $z^{d^{j}}=\Omega$ ), among which at least one is not a zero or pole of $R\left(z^{d^{j-1}}\right) \cdot \ldots \cdot R(z)$. Thus, on every circle $|z|=|\omega|^{1 / d^{j}}$ (or $|z|=|\Omega|^{1 / d^{j}}$ ), we have a zero or a pole of $r$ contradicting the rationality of $r$.

The central role in the proof of Theorem 1.4 will be played by the following lemma.

Lemma 3.3. Assume that $p_{1}(z), \ldots, p_{n}(z)$ satisfy the assumptions of Theorem 1.4. If $P$ is irreducible and depends on at least one of the $x_{i}, y_{i}$, then an identity

$$
T(z) P\left(z, x_{1}, y_{1}, \ldots, x_{n}, y_{n}\right)=Q\left(z, x_{1}, y_{1}, \ldots, x_{n}, y_{n}\right)
$$

with a polynomial $T(z)$ is impossible.

Proof. Assume that (11) holds with some polynomial $T(z)$ (we shall see $T(z) \neq 0$ from (15) below). Then $T \mid Q$, and therefore Lemma 3.1 yields

$$
T(z) \mid p_{\underline{m}_{n}}\left(z^{d}\right) \prod_{i=1}^{n} U_{i}(z)^{M}
$$

for all $\underline{m}_{n}$, where $M(\geq 1)$ was introduced after $(7)$. Since $P$ is irreducible, this gives the important information

$$
T(z) \mid \prod_{i=1}^{n} U_{i}(z)^{M} .
$$

For the rest of the proof, we write $P$ in the shape

$$
P\left(z, x_{1}, y_{1}, \ldots, x_{n}, y_{n}\right)=\sum_{j=0}^{M} \sum_{\left|\underline{m}_{n}\right|=j} p_{\underline{m}_{n}}(z) \prod_{i=1}^{n} x_{i}^{\ell_{i}} y_{i}^{k_{i}}=: \sum_{j=0}^{M} P_{j}\left(z, x_{1}, y_{1}, \ldots, x_{n}, y_{n}\right),
$$

as after (7). Then correspondingly

$$
Q\left(z, x_{1}, y_{1}, \ldots, x_{n}, y_{n}\right)=\sum_{j=0}^{M} \sum_{\left|\underline{m}_{n}\right|=j} Q_{\underline{m}_{n}}\left(z, x_{1}, y_{1}, \ldots, x_{n}, y_{n}\right)=: \sum_{j=0}^{M} Q_{j}\left(z, x_{1}, y_{1}, \ldots, x_{n}, y_{n}\right),
$$

and here $Q_{\underline{m}_{n}}\left(z, x_{1}, y_{1}, \ldots, x_{n}, y_{n}\right)$ is defined as in (8). By (11),

$$
T(z) P_{j}\left(z, x_{1}, y_{1}, \ldots, x_{n}, y_{n}\right)=Q_{j}\left(z, x_{1}, y_{1}, \ldots, x_{n}, y_{n}\right) \quad(j=0, \ldots, M) .
$$

To study the leading terms, we write

$$
P_{M}\left(z, x_{1}, y_{1}, \ldots, x_{n}, y_{n}\right)=\sum_{k=0}^{M} \sum_{\left|\underline{m}_{n-1}\right|=M-k} \sum_{\ell_{n}+k_{n}=k} p_{\underline{m}_{n}}(z) \prod_{i=1}^{n} x_{i}^{\ell_{i}} y_{i}^{k_{i}}=: \sum_{k=0}^{M} P_{M, k}\left(z, x_{1}, y_{1}, \ldots, x_{n}, y_{n}\right)
$$


and

$$
Q_{M}\left(z, x_{1}, y_{1}, \ldots, x_{n}, y_{n}\right)=\sum_{k=0}^{M} Q_{M, k}\left(z, x_{1}, y_{1}, \ldots, x_{n}, y_{n}\right)
$$

with

$$
Q_{M, k}\left(z, x_{1}, y_{1}, \ldots, x_{n}, y_{n}\right):=\sum_{\left|\underline{m}_{n-1}\right|=M-k} \sum_{\ell_{n}+k_{n}=k} Q_{\underline{m}_{n}}\left(z, x_{1}, y_{1}, \ldots, x_{n}, y_{n}\right) .
$$

Let $K_{n}$ be the maximal value of $k$ such that at least one $p_{\underline{m}_{n}}(z) \neq 0$ in $P_{M, k}$. Then $P_{M, k}=0$ and $Q_{M, k}=0$ for all $k>K_{n}$, and moreover

$$
\begin{aligned}
P_{M, K_{n}}\left(z, x_{1}, y_{1}, \ldots, x_{n}, y_{n}\right)=\sum_{k_{n}=0}^{K_{n}} & \sum_{k=0}^{M-K_{n}} \sum_{\left|\underline{m}_{n-2}\right|=M-K_{n}-k} \sum_{\ell_{n-1}+k_{n-1}=k} p_{\underline{m}_{n-1}, K_{n}-k_{n}, k_{n}}(z) \\
& \times\left(\prod_{i=1}^{n-1} x_{i}^{\ell_{i}} y_{i}^{k_{i}}\right) x_{n}^{K_{n}-k_{n}} y_{n}^{k_{n}}=: \sum_{k=0}^{M-K_{n}} P_{M, K_{n}, k}\left(z, x_{1}, y_{1}, \ldots, x_{n}, y_{n}\right) .
\end{aligned}
$$

Let now $K_{n-1}$ be the maximal $k$ such that at least one $p_{\underline{m}_{n-1}, K_{n}-k_{n}, k_{n}}(z) \neq 0$ in $P_{M, K_{n}, k}$. Then

$$
\begin{aligned}
P_{M, K_{n}, K_{n-1}}\left(z, x_{1}, y_{1}, \ldots, x_{n}, y_{n}\right)=\sum_{k_{n}=0}^{K_{n}} \sum_{k_{n-1}=0}^{K_{n-1}} \sum_{\left|\underline{m}_{n-2}\right|=} \sum_{M-K_{n}-K_{n-1}} p_{\underline{m}_{n-2}, K_{n-1}-k_{n-1}, k_{n-1}, K_{n}-k_{n}, k_{n}}(z) \\
\times\left(\prod_{i=1}^{n-2} x_{i}^{\ell_{i}} y_{i}^{k_{i}}\right) x_{n-1}^{K_{n-1}-k_{n-1}} y_{n-1}^{k_{n-1}} x_{n}^{K_{n}-k_{n}} y_{n}^{k_{n}} .
\end{aligned}
$$

By continuing in this way, we construct a non-zero polynomial

$$
P_{M, K_{n}, \ldots, K_{1}}\left(z, x_{1}, y_{1}, \ldots, x_{n}, y_{n}\right)=\sum_{k_{n}=0}^{K_{n}} \ldots \sum_{k_{1}=0}^{K_{1}} p_{K_{1}-k_{1}, k_{1}, \ldots, K_{n}-k_{n}, k_{n}}(z) \prod_{i=1}^{n} x_{i}^{K_{i}-k_{i}} y_{i}^{k_{i}},
$$

where $K_{1}+\ldots+K_{n}=M$ and, by $(13)$,

$$
T(z) P_{M, K_{n}, \ldots, K_{1}}\left(z, x_{1}, y_{1}, \ldots, x_{n}, y_{n}\right)=Q_{M, K_{n}, \ldots, K_{1}}\left(z, x_{1}, y_{1}, \ldots, x_{n}, y_{n}\right):=
$$

$$
\sum_{k_{n}=0}^{K_{n}} \ldots \sum_{k_{1}=0}^{K_{1}} p_{K_{1}-k_{1}, k_{1}, \ldots, K_{n}-k_{n}, k_{n}}\left(z^{d}\right) \prod_{i=1}^{n}\left(U_{i}(z)^{M-K_{i}}\left(d z^{d-1} p_{i}(z) x_{i}\right)^{K_{i}-k_{i}}\left(-p_{i}^{\prime}(z) x_{i}+p_{i}(z) y_{i}\right)^{k_{i}}\right) .
$$

Let now $\hat{k}_{1}$ denote the maximal value of $k_{1}$ such that $p_{K_{1}-k_{1}, k_{1}, \ldots, K_{n}-k_{n}, k_{n}}(z) \neq 0$ for some $k_{2}, \ldots, k_{n}$, and let then $\hat{k}_{2}$ be the maximal value $k_{2}$ such that $p_{K_{1}-\hat{k}_{1}, \hat{k}_{1}, K_{2}-k_{2}, k_{2}, \ldots, K_{n}-k_{n}, k_{n}}(z) \neq 0$ for some $k_{3}, \ldots, k_{n}$. By continuing analogously, we fix also $\hat{k}_{3}, \ldots, \hat{k}_{n}$. This construction means, in particular, that $p_{K_{1}-\hat{k}_{1}, \hat{k}_{1}, \ldots, K_{n-1}-\hat{k}_{n-1}, \hat{k}_{n-1}, K_{n}-k_{n}, k_{n}}(z)=0$ for all $k_{n}>\hat{k}_{n}$. By $(14)$,

$$
T(z) p_{K_{1}-\hat{k}_{1}, \hat{k}_{1}, \ldots, K_{n}-\hat{k}_{n}, \hat{k}_{n}}(z)=p_{K_{1}-\hat{k}_{1}, \hat{k}_{1}, \ldots, K_{n}-\hat{k}_{n}, \hat{k}_{n}}\left(z^{d}\right) \prod_{i=1}^{n}\left(U_{i}(z)^{M-K_{i}}\left(d z^{d-1}\right)^{K_{i}-\hat{k}_{i}} p_{i}(z)^{K_{i}}\right)
$$

holds implying $T(z) \neq 0$. By (12), we know that possible zeros of $T(z)$ having absolute value $\neq 0,1$ are the zeros of $\prod_{i=1}^{n} v_{i}(z)$, which we denote by $\alpha_{\mu}(\mu=1, \ldots, N)$. If some $\alpha_{\mu}$ 's are zeros or poles of $\left(\prod_{i=1}^{n} v_{i}(z)^{2 M-K_{i}}\right) / T(z)$, let $\omega$ denote such $\alpha_{\mu}$ with minimal absolute value and $\Omega$ with maximal absolute value. Then $|\omega|<1$ or $|\Omega|>1$, and, by the assumptions of Lemma 3.3, we get a contradiction from Lemma 3.2. Thus

$$
T(z)=c_{0}(z) \prod_{i=1}^{n} v_{i}(z)^{2 M-K_{i}}
$$


where $c_{0}(z)$ (as also $c_{1}(z), c_{2}(z), \ldots$ later) is a non-zero polynomial with possible zeros of absolute value 0 or 1 . Further, by (15),

$$
c_{0}(z) p_{K_{1}-\hat{k}_{1}, \hat{k}_{1}, \ldots, K_{n}-\hat{k}_{n}, \hat{k}_{n}}(z)=p_{K_{1}-\hat{k}_{1}, \hat{k}_{1}, \ldots, K_{n}-\hat{k}_{n}, \hat{k}_{n}}\left(z^{d}\right) c_{1}(z) .
$$

If $\hat{k}_{n} \geq 1$, then, by comparing the coefficients of $\left(\prod_{i=1}^{n-1} x_{i}^{K_{i}-\hat{k}_{i}} y_{i}^{\hat{k}_{i}}\right) x_{n}^{K_{n}-\hat{k}_{n}+1} y_{n}^{\hat{k}_{n}-1}$ on both sides of (14) and denoting $p_{i}(z)=u_{i}(z) v_{i}(z)(i=1, \ldots, n)$, we get

$$
\begin{aligned}
& T(z) p_{K_{1}-\hat{k}_{1}, \hat{k}_{1}, \ldots, K_{n-1}-\hat{k}_{n-1}, \hat{k}_{n-1}, K_{n}-\hat{k}_{n}+1, \hat{k}_{n}-1}(z) \\
& \quad=p_{K_{1}-\hat{k}_{1}, \hat{k}_{1}, \ldots, K_{n-1}-\hat{k}_{n-1}, \hat{k}_{n-1}, K_{n}-\hat{k}_{n}+1, \hat{k}_{n}-1}\left(z^{d}\right) c_{2}(z) \prod_{i=1}^{n} v_{i}(z)^{2 M-K_{i}} \\
& \quad+p_{K_{1}-\hat{k}_{1}, \hat{k}_{1}, \ldots, K_{n}-\hat{k}_{n}, \hat{k}_{n}}\left(z^{d}\right) c_{3}(z)\left(\prod_{i=1}^{n-1} v_{i}(z)^{2 M-K_{i}}\right) v_{n}(z)^{2 M-K_{n}-1}\left(u_{n}^{\prime}(z) v_{n}(z)+u_{n}(z) v_{n}^{\prime}(z)\right) .
\end{aligned}
$$

This equation implies $p_{K_{1}-\hat{k}_{1}, \hat{k}_{1}, \ldots, K_{n}-\hat{k}_{n}, \hat{k}_{n}}\left(\alpha^{d}\right)=0$ if $\alpha$ is a zero of $v_{n}(z)$. But then, by $(17)$, $p_{K_{1}-\hat{k}_{1}, \hat{k}_{1}, \ldots, K_{n}-\hat{k}_{n}, \hat{k}_{n}}\left(\alpha^{d^{j}}\right)=0$ for all $j \geq 1$, a contradiction. Thus $\hat{k}_{n}=0$. In particular, in the case $n=1$, the polynomial $P_{M}\left(z, x_{1}, y_{1}\right)$ reduces to $p_{M, 0}(z) x_{1}^{M}$, and, by the irreducibility of $P$, there must be some $j<M$ with $P_{j} \neq 0$.

Assume that $P_{j} \neq 0$ with some $j<M$, and denote by $J$ the maximal $j$ with this property. We then write $P_{J}$ as $P_{M}$ above and use $K_{1}^{*}, \ldots, K_{n}^{*}, k_{1}^{*}, \ldots, k_{n}^{*}$ in similar roles as $K_{1}, \ldots, K_{n}, \hat{k}_{1}, \ldots, \hat{k}_{n}$ before. Then, similarly to (15),

$$
T(z) p_{K_{1}^{*}-k_{1}^{*}, k_{1}^{*}, \ldots, K_{n}^{*}-k_{n}^{*}, k_{n}^{*}}(z)=p_{K_{1}^{*}-k_{1}^{*}, k_{1}^{*}, \ldots, K_{n}^{*}-k_{n}^{*}, k_{n}^{*}}\left(z^{d}\right) \prod_{i=1}^{n}\left(U_{i}(z)^{M-K_{i}^{*}}\left(d z^{d-1}\right)^{K_{i}^{*}-k_{i}^{*}} p_{i}(z)^{K_{i}^{*}}\right)
$$

Since $K_{1}^{*}+\cdots+K_{n}^{*}=J<M,(16)$ and Lemma 3.2 give a contradiction as above. Thus $P_{j}=0$ for all $j<M$. In particular, this proves Lemma 3.3 in the case $n=1$.

For the rest of the proof, we make the inductive assumption that Lemma 3.3 holds, if $n(\geq 2)$ is replaced by $n-1$. To complete our proof, we next show that

$$
P\left(z, x_{1}, y_{1}, \ldots, x_{n}, y_{n}\right)=P_{M, K_{n}, \ldots, K_{1}}\left(z, x_{1}, y_{1}, \ldots, x_{n}, y_{n}\right) .
$$

Let us assume that there exists some $k<K_{n}$ such that at least one $p_{\underline{m}_{n}}(z)$ in $P_{M, k}$ is non-zero, and denote by $\widetilde{K}_{n}\left(<K_{n}\right)$ the maximal $k$ with this property. We then define $\widetilde{K}_{1}, \ldots, \widetilde{K}_{n}$ and $\widetilde{k}_{1}, \ldots, \widetilde{k}_{n}$ similarly to $K_{1}, \ldots, K_{n}$ and $\hat{k}_{1}, \ldots, \hat{k}_{n}$ above. Analogously to (15) we have

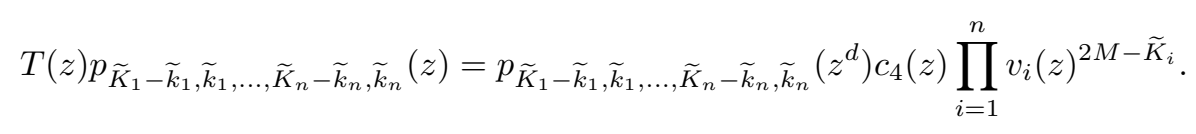

But then (16) gives

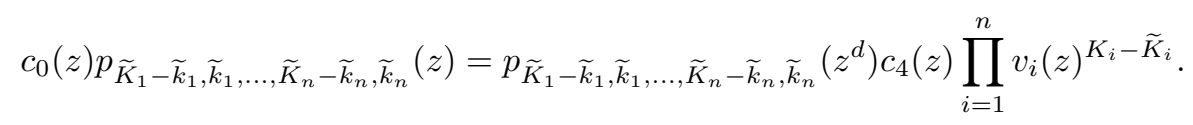

Since $K_{n}>\widetilde{K}_{n}$, this leads to a contradiction, by Lemma 3.2. Therefore

$$
P\left(z, x_{1}, y_{1}, \ldots, x_{n}, y_{n}\right)=P_{M, K_{n}}\left(z, x_{1}, y_{1}, \ldots, x_{n}, y_{n}\right)
$$

If there exists some $k<K_{n-1}$ such that at least one $p_{\underline{m}_{n}}(z)$ in $P_{M, K_{n}, k}$ is non-zero, then let $\widetilde{K}_{n-1}\left(<K_{n-1}\right)$ denote the maximal such $k$. Again we define new $\widetilde{K}_{1}, \ldots, \widetilde{K}_{n-1}\left(<K_{n-1}\right), \widetilde{K}_{n}=K_{n}$ 
and $\widetilde{k}_{1}, \ldots, \widetilde{k}_{n}$ in a similar way as $K_{1}, \ldots, K_{n}$ and $\hat{k}_{1}, \ldots, \hat{k}_{n}$ above. This leads once more to (19), which gives a contradiction, by Lemma 3.2 , since $K_{n-1}>\widetilde{K}_{n-1}$. Thus

$$
P\left(z, x_{1}, y_{1}, \ldots, x_{n}, y_{n}\right)=P_{M, K_{n}, K_{n-1}}\left(z, x_{1}, y_{1}, \ldots, x_{n}, y_{n}\right) .
$$

By continuing in this way, we obtain equation (18).

If $p_{K_{1}-k_{1}, k_{1}, \ldots, K_{n}-k_{n}, k_{n}}(z)=0$ for all $\left(k_{1}, \ldots, k_{n}\right)$ with $0 \leq k_{1} \leq \hat{k}_{1}, 0 \leq k_{i} \leq K_{i}(1<i<n), k_{n} \geq 1$, then $P$ has a factor $x_{n}^{K_{n}}$, so $K_{n}=0$ by the irreducibility of $P$, and

$$
P\left(z, x_{1}, y_{1}, \ldots, x_{n}, y_{n}\right)=\sum_{k_{n-1}=0}^{K_{n-1}} \ldots \sum_{k_{1}=0}^{K_{1}} p_{K_{1}-k_{1}, k_{1}, \ldots, K_{n-1}-k_{n-1}, k_{n-1}, 0,0}(z) \prod_{i=1}^{n-1} x_{i}^{K_{i}-k_{i}} y_{i}^{k_{i}} .
$$

The induction hypothesis gives now a contradiction. Thus, there must exist a non-zero polynomial $p_{K_{1}-k_{1}, k_{1}, \ldots, K_{n}-k_{n}, k_{n}}(z)$ with $k_{n} \geq 1$. Let $\widetilde{k}_{1}$ be the maximal $k_{1}$ with this property, and after that, let $\widetilde{k}_{2}$ be the maximal $k_{2}$ with $p_{K_{1}-\widetilde{k}_{1}, \widetilde{k}_{1}, K_{2}-k_{2}, k_{2}, \ldots, K_{n}-k_{n}, k_{n}}(z) \neq 0, k_{n} \geq 1$. We continue to define $\widetilde{k}_{3}, \ldots, \widetilde{k}_{n}$ in this way. Then $p_{K_{1}-\widetilde{k}_{1}, \widetilde{k}_{1}, \ldots, K_{n}-\widetilde{k}_{n}, \widetilde{k}_{n}}(z) \neq 0, \widetilde{k}_{n} \geq 1$, and $p_{K_{1}-\widetilde{k}_{1}, \widetilde{k}_{1}, \ldots, K_{n-1}-\widetilde{k}_{n-1}, \widetilde{k}_{n-1}, K_{n}-k_{n}, k_{n}}(z)=0$ for all $k_{n}>\widetilde{k}_{n}$. Similarly to (15),

$$
T(z) p_{K_{1}-\widetilde{k}_{1}, \widetilde{k}_{1}, \ldots, K_{n}-\widetilde{k}_{n}, \widetilde{k}_{n}}(z)=p_{K_{1}-\widetilde{k}_{1}, \widetilde{k}_{1}, \ldots, K_{n}-\widetilde{k}_{n}, \widetilde{k}_{n}}\left(z^{d}\right) \prod_{i=1}^{n}\left(U_{i}(z)^{M-K_{i}}\left(d z^{d-1}\right)^{K_{i}-\widetilde{k}_{i}} p_{i}(z)^{K_{i}}\right) .
$$

By (16),

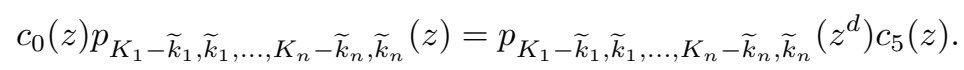

If $\widetilde{k}_{n}>1$, then we compare the coefficients of $\left(\prod_{i=1}^{n-1} x_{i}^{K_{i}-\widetilde{k}_{i}} y_{i}^{\widetilde{k}_{i}}\right) x_{n}^{K_{n}-\widetilde{k}_{n}+1} y_{n}^{\widetilde{k}_{n}-1}$ on both sides of (14) and obtain

$$
\begin{aligned}
& T(z) p_{K_{1}-\widetilde{k}_{1}, \widetilde{k}_{1}, \ldots, K_{n-1}-\widetilde{k}_{n-1}, \widetilde{k}_{n-1}, K_{n}-\widetilde{k}_{n}+1, \widetilde{k}_{n}-1}(z)= \\
& p_{K_{1}-\widetilde{k}_{1}, \widetilde{k}_{1}, \ldots, K_{n-1}-\widetilde{k}_{n-1}, \widetilde{k}_{n-1}, K_{n}-\widetilde{k}_{n}+1, \widetilde{k}_{n}-1}\left(z^{d}\right) c_{6}(z) \prod_{i=1}^{n} v_{i}(z)^{2 M-K_{i}}+ \\
& p_{K_{1}-\widetilde{k}_{1}, \widetilde{k}_{1}, \ldots, K_{n-1}-\widetilde{k}_{n-1}, \widetilde{k}_{n-1}, K_{n}-\widetilde{k}_{n}, \widetilde{k}_{n}}\left(z^{d}\right) c_{7}(z)\left(\prod_{i=1}^{n-1} v_{i}(z)^{2 M-K_{i}}\right) v_{n}(z)^{2 M-K_{n}-1}\left(u_{n}^{\prime}(z) v_{n}(z)+u_{n}(z) v_{n}^{\prime}(z)\right) .
\end{aligned}
$$

This implies $p_{K_{1}-\widetilde{k}_{1}, \widetilde{k}_{1}, \ldots, K_{n-1}-\widetilde{k}_{n-1}, \widetilde{k}_{n-1}, K_{n}-\widetilde{k}_{n}, \widetilde{k}_{n}}\left(\alpha^{d}\right)=0$ for all zeros $\alpha$ of $v_{n}(z)$ and, as above, this leads to a contradiction, by (20).

To consider the case $\widetilde{k}_{n}=1$, we compare the coefficient of $\left(\prod_{i=1}^{n-1} x_{i}^{K_{i}-\widetilde{k}_{i}} y_{i}^{\widetilde{k}_{i}}\right) x_{n}^{K_{n}}$ on both sides of (14). Each term

$$
p_{K_{1}-k_{1}, k_{1}, \ldots, K_{n-1}-k_{n-1}, k_{n-1}, K_{n}, 0}(z) \prod_{i=1}^{n} x_{i}^{K_{i}-k_{i}} y_{i}^{k_{i}}
$$

with $\left(k_{1}, \ldots, k_{n-1}\right)$ satisfying $\widetilde{k}_{1} \leq k_{1} \leq \hat{k}_{1}$ and $\widetilde{k}_{i} \leq k_{i} \leq K_{i}$ for $i=2, \ldots, n-1$ gives a contribution

$$
\begin{aligned}
& p_{K_{1}-k_{1}, k_{1}, \ldots, K_{n-1}-k_{n-1}, k_{n-1}, K_{n}, 0}\left(z^{d}\right) c_{8, k_{i}}(z) \\
& \quad \times\left(\prod_{i=1}^{n-1} v_{i}(z)^{2 M-K_{i}-k_{i}+\widetilde{k}_{i}}\left(u_{i}^{\prime}(z) v_{i}(z)+u_{i}(z) v_{i}^{\prime}(z)\right)^{k_{i}-\widetilde{k}_{i}} x_{i}^{K_{i}-\widetilde{k}_{i}} y_{i}^{\widetilde{k}_{i}}\right) v_{n}(z)^{2 M-K_{n}} x_{n}^{K_{n}}
\end{aligned}
$$


to the $Q$-polynomial. Therefore

$$
\begin{aligned}
& T(z) p_{K_{1}-\widetilde{k}_{1}, \widetilde{k}_{1}, \ldots, K_{n-1}-\widetilde{k}_{n-1}, \widetilde{k}_{n-1}, K_{n}, 0}(z)= \\
& \sum_{k_{1}=\widetilde{k}_{1}} \sum_{k_{2}=\widetilde{k}_{2}}^{K_{2}} \ldots \sum_{k_{n-1}=\widetilde{k}_{n-1}}^{K_{n-1}} p_{K_{1}-k_{1}, k_{1}, \ldots, K_{n-1}-k_{n-1}, k_{n-1}, K_{n}, 0}\left(z^{d}\right) c_{8, k_{i}}(z) \times \\
& \quad\left(\prod_{i=1}^{n-1} v_{i}(z)^{2 M-K_{i}-k_{i}+\widetilde{k}_{i}}\left(u_{i}^{\prime}(z) v_{i}(z)+u_{i}(z) v_{i}^{\prime}(z)\right)^{k_{i}-\widetilde{k}_{i}}\right) v_{n}(z)^{2 M-K_{n}}+ \\
& p_{K_{1}-\widetilde{k}_{1}, \widetilde{k}_{1}, \ldots, K_{n-1}-\widetilde{k}_{n-1}, \widetilde{k}_{n-1}, K_{n}-1,1}\left(z^{d}\right) c_{9}(z)\left(\prod_{i=1}^{n-1} v_{i}(z)^{2 M-K_{i}}\right) v_{n}(z)^{2 M-K_{n}-1}\left(u_{n}^{\prime}(z) v_{n}(z)+u_{n}(z) v_{n}^{\prime}(z)\right) .
\end{aligned}
$$

By using (16), we have $p_{K_{1}-\widetilde{k}_{1}, \widetilde{k}_{1}, \ldots, K_{n-1}-\widetilde{k}_{n-1}, \widetilde{k}_{n-1}, K_{n-1,1}}\left(\alpha^{d}\right)=0$ for all zeros $\alpha$ of $v_{n}(z)$, and a contradiction follows from $(20)$, where now $\widetilde{k}_{n}=1$. This finally proves Lemma 3.3 .

Proof of Theorem 1.4. The functions $F_{1}(z)$ and $F_{1}^{\prime}(z)$ are algebraically independent by Theorem 1.1. Assume now that Theorem 1.4 holds if $n(\geq 2)$ is replaced by $n-1$, whence the functions $F_{i}(z), F_{i}^{\prime}(z)(i=$ $1, \ldots, n-1)$ are algebraically independent. If these functions and $F_{n}(z)$ were algebraically dependent, then there exists an irreducible polynomial $P\left(z, x_{1}, y_{1}, \ldots, x_{n-1}, y_{n-1}, x_{n}\right) \neq 0$ such that

$$
P\left(z, F_{1}(z), F_{1}^{\prime}(z), \ldots, F_{n-1}(z), F_{n-1}^{\prime}(z), F_{n}(z)\right)=0 .
$$

By our induction hypothesis, we have $\operatorname{deg}_{x_{n}} P \geq 1$. From the above proof we see that the corresponding polynomial $Q \neq 0$, and this $Q$ must be divisible by $P$, since otherwise the resultant of $P$ and $Q$ with respect to $x_{n}$ gives an algebraic dependence relation of $F_{i}(z), F_{i}^{\prime}(z)(i=$ $1, \ldots, n-1)$. Thus, there exists a polynomial $T(z) \neq 0$ such that $T(z) P\left(z, x_{1}, y_{1}, \ldots, x_{n-1}, y_{n-1}, x_{n}\right)=$ $Q\left(z, x_{1}, y_{1}, \ldots, x_{n-1}, y_{n-1}, x_{n}\right)$, but this is impossible, by (a special case of) Lemma 3.3 . This proves the algebraic independence of $F_{i}(z), F_{i}^{\prime}(z)(i=1, \ldots, n-1)$ and $F_{n}(z)$.

If the $2 n$ functions $F_{i}(z), F_{i}^{\prime}(z)(i=1, \ldots, n)$ were algebraically dependent, then there would be an irreducible polynomial $P\left(z, x_{1}, y_{1}, \ldots, x_{n}, y_{n}\right) \neq 0$ such that

$$
P\left(z, F_{1}(z), F_{1}^{\prime}(z), \ldots, F_{n}(z), F_{n}^{\prime}(z)\right)=0
$$

and necessarily $\operatorname{deg}_{y_{n}} P \geq 1$. Now Lemma 3.3 gives a contradiction as above proving Theorem 1.4.

4. Proof of Theorem 1.11. Our proof relies on the subsequent criterion of Kubota [7, Proposition 3] (see also [9, Theorem 3.5]) to be quoted here in a very particular version, which suffices for our purposes.

LEMMA 4.1. Suppose that the series $f_{1}, \ldots, f_{k} \in \mathbb{C}[[z]] \backslash\{0\}$ converge on $\mathbb{D}$ and satisfy the functional equations

$$
f_{i}\left(z^{d}\right)=b_{i}(z) f_{i}(z) \quad(i=1, \ldots, k)
$$

with all $b_{i} \in \mathbb{C}(z) \backslash\{0\}$ fulfilling the condition that, for no $\left(e_{1}, \ldots, e_{k}\right) \in \mathbb{Z}^{k} \backslash\{\underline{0}\}$, the functional equation

$$
r\left(z^{d}\right)=r(z) \prod_{i=1}^{k} b_{i}(z)^{e_{i}}
$$

has a solution $r \in \mathbb{C}(z) \backslash\{0\}$. Then the functions $f_{1}, \ldots, f_{k}$ are algebraically independent over $\mathbb{C}(z)$.

Proof of Theorem 1.11. We apply Lemma 4.1 with $k=n+m, f_{i}(z)=F_{i}(z)(i=1, \ldots, n)$ and $f_{n+u}(z)=\mathcal{F}_{\ell_{u}}(z)(u=1, \ldots, m)$ satisfying $\operatorname{gcd}\left(d, \ell_{u}\right)=1$. Now $b_{i}(z)=1 / p_{i}(z)(i=1, \ldots, n), b_{n+u}(z)=$ 
$1 / \Phi_{\ell_{u}}(z)(u=1, \ldots, m)$, and we must show, for any $\left(e_{1}, \ldots, e_{k}\right) \in \mathbb{Z}^{k} \backslash\{\underline{0}\}$, that the equation

$$
r(z)=r\left(z^{d}\right)\left(\prod_{i=1}^{n} p_{i}(z)^{e_{i}}\right)\left(\prod_{u=1}^{m} \Phi_{\ell_{u}}(z)^{e_{n+u}}\right)
$$

has no non-trivial rational solution $r$.

To consider first the case $e_{1}=\cdots=e_{n}=0$, we iterate (21) $j$ times and obtain

$$
r(z)=r\left(z^{d^{j}}\right) \prod_{u=1}^{m}\left(\prod_{i=0}^{j-1} \Phi_{\ell_{u}}\left(z^{d^{i}}\right)\right)^{e_{n+u}} .
$$

Letting $j \rightarrow \infty$ and noting $r(0) \neq 0, \infty$, this formula leads to $r(z)=r(0) \prod_{u=1}^{m} \mathcal{F}_{\ell_{u}}(z)^{e_{n+u}}$, a contradiction, since the functions $\mathcal{F}_{\ell_{u}}(z)$ are algebraically independent over $\mathbb{C}(z)$, by [3, Theorem 1.3].

To consider the second subcase $\left(e_{1}, \ldots, e_{n}\right) \neq \underline{0}$ of $\left(e_{1}, \ldots, e_{k}\right)$, we apply Lemma 3.2 with

$$
R(z):=\left(\prod_{i=1}^{n} p_{i}(z)^{e_{i}}\right)\left(\prod_{u=1}^{m} \Phi_{\ell_{u}}(z)^{e_{n+u}}\right)
$$

being the factor appearing in the right-hand side of (21). By the assumption in Theorem 1.11, Lemma 3.2 says that $(21)$ does not have a rational solution $r(z) \neq 0$. Then the validity of Theorem 1.11 follows from Lemma 4.1.

\section{References}

[1] R. Brent, M. Coons and W. Zudilin, Algebraic independence of Mahler functions via radial asymptotics, Int. Math. Res. Notices, 2016 (2) (2016), 571-603.

[2] P. Bundschuh, Algebraic independence of infinite products and their derivatives, in: J. M. Borwein et al., Number Theory and Related Fields, Springer Proc. Math. 43 (2013), 143-156.

[3] P. Bundschuh and K. Väänänen, Arithmetic properties of infinite products of cyclotomic polynomials, Bull. Aust. Math. Soc. 93 (2016), 375-387.

[4] P. Bundschuh and K. Väänänen, Note on the Stern-Brocot sequence, some relatives, and their generating power series, J. Théor. Nombres Bordeaux (to appear).

[5] T. Dreyfus, C. Hardoun and J. Roques, Hypertranscendence of solutions of Mahler equations, Preprint, 2015, available at arXiv:1507.03361.

[6] W. Duke and H.N. Nguyen, Infinite products of cyclotomic polynomials, Bull. Aust. Math. Soc. 91 (2015), 400-411.

[7] K. K. Kubota, On the algebraic independence of holomorphic solutions of certain functional equations and their values, Math. Ann. 227 (1977), 9-50.

[8] Ke. Nishioka, A note on differentially algebraic solutions of first order linear difference equations, Aequationes Math. 27 (1984), 32-48.

[9] K. Nishioka, Mahler Functions and Transcendence, Lecture Notes in Math. 1631, Springer, Berlin, 1996.

Peter Bundschuh

Mathematisches Institut

Universität zu Köln

Weyertal 86-90

50931 Köln, Germany

E-mail: pb@math.uni-koeln.de
Keijo Väänänen

Department of Mathematical Sciences

University of Oulu

P. O. Box 3000

90014 Oulu, Finland

E-mail: kvaanane@sun3.oulu.fi 